\title{
GENOMICS
}

\section{Counting short tandem repeats}

A method using CRISPR-Cas technology, nanopore sequencing and bioinformatics enables quantification of short tandem repeats with high accuracy.

S hort tandem repeats (STRs) are nucleotide motifs of 2-6 base pairs in length and are found in repeat units throughout the human genome. The number of STR expansions can be polymorphic, but expansion beyond a normal threshold is associated with genetic disorders such as fragile X syndrome and amyotrophic lateral sclerosis (ALS). Southern blotting or PCRbased gel electrophoresis have traditionally been the gold standard to assess STR status, but these are labor-intensive. Highthroughput or nanopore-based sequencing methods combined with advanced bioinformatics base-calling algorithms offer a cost-effective alternative to characterize and assess STRs. Despite these advances, characterization of STRs remains limited to resolve expansion of repeats greater than 32 owing to issues with signal processing and target enrichment.
Franz-Josef Müller and his colleagues sought a new strategy to address these concerns to enable nanopore-sequencingmediated quantitation of STRs. They used the CRISPR-Cas12a or Cas9 ribonucleoprotein to enrich for STRs twofold through promoting double-strand breaks near the repeat site. They ligated the resulting fragments to nanopore sequencing adaptors to increase nanopore sequencing depth. Müller and colleagues then developed an algorithm called short tandem repeat identification, quantification and evaluation (STRique) using hidden Markov model-based repeat counting mechanism and raw signal alignment to assess STRs from nanopore sequencing reads. Analysis of plasmids and bacterial artificial chromosomes containing known repeat lengths matched gel electrophoresis analysis with higher accuracy relative to existing base calling algorithms.
Müller and colleagues used STRique to characterize STRs from human induced pluripotent stem cell line derived from an individual with ALS, and showed that pathogenic STR expansions were correlated with increased CpG methylation at the promoter whereas cells from patients without ALS did not exhibit alterations in CpG levels. Overall, the STRique method offers precise quantification of STRs, which may help elucidate connections between repeat expansion and epigenetic regulation.

\section{Grant Miura}

Published online: 6 January 2020

https://oi.org/10.1038/s41592-019-0724-0

Research paper

Giesselmann, P. et al. Analysis of short tandem repeat expansions and their methylation state with nanopore sequencing. Nat. Biotechnol. 37, 1478-1481 (2019).

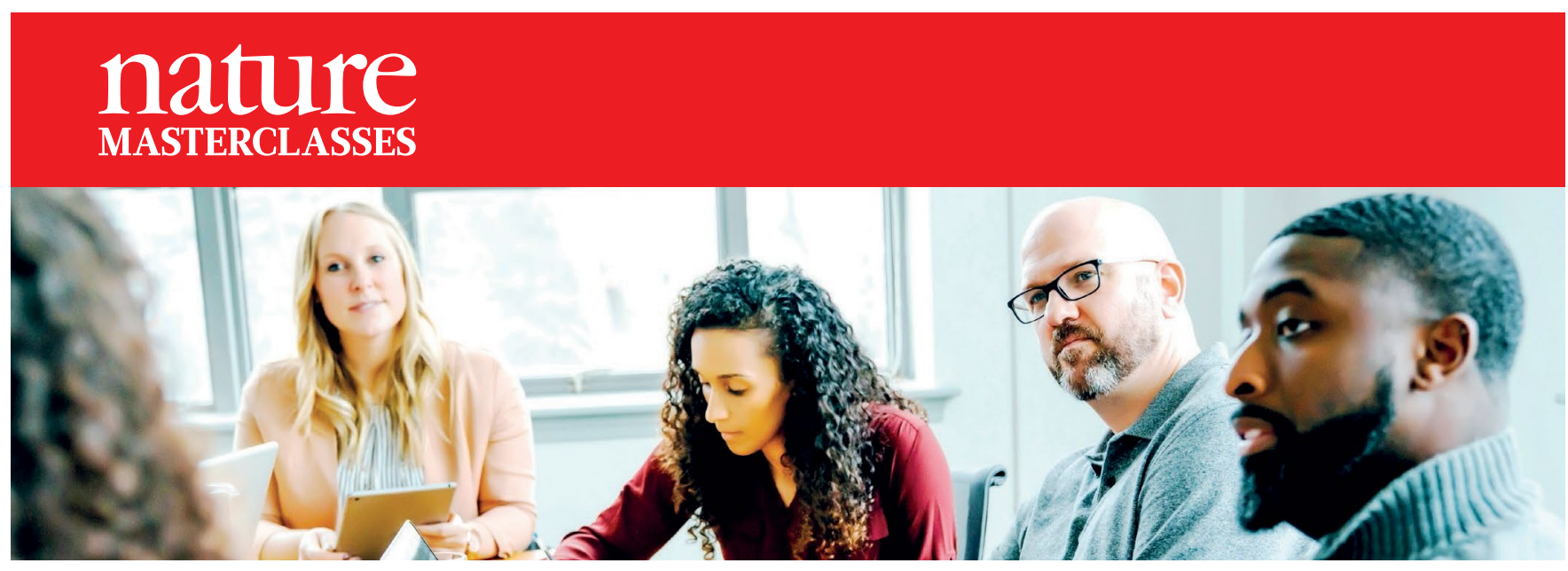

\section{Workshops in Scientific Writing and Publishing}

\author{
Delivered by Nature Research journal editors and hosted at institutions worldwide, \\ researchers learn how to turn great science into great papers.
}

Find out more at masterclasses.nature.com 